\title{
A Molecular Communication Model of Exosome-mediated Brain Drug Delivery
}

\author{
Mladen Veletić \\ Oslo University Hospital \\ Oslo, Norway \\ mlavel@rr-research.no \\ Ilangko Balasingham \\ Oslo University Hospital//Norw. Uni. of Science and Tech. \\ Oslo//Trondheim, Norway \\ ilangko.balasingham@ntnu.no
}

\author{
Michael Taynnan Barros \\ Waterford Institute of Technology \\ Waterford, Ireland \\ mbarros@tssg.org \\ Sasitharan Balasubramaniam
Waterford Institute of Technology
Waterford, Ireland
sasib@tssg.org
}

ACM Reference Format:

Mladen Veletić, Michael Taynnan Barros, Ilangko Balasingham, and Sasitharan Balasubramaniam. 2019. A Molecular Communication Model of Exosome-mediated Brain Drug Delivery. In The Sixth Annual ACM International Conference on Nanoscale Computing and Communication (NANOCOM '19), September 25-27, 2019, Dublin, Ireland. ACM, New York, NY, USA, 7 pages. https://doi.org/10.1145/3345312.3345478

\section{INTRODUCTION}

Glioblastoma Multiforme is the most prevalent and devastating brain disease whose treatment have the lowest success rates compared to other therapeutic cancer technologies [9]. The development of brain drug delivery systems for this type of cancer is very challenging because of side effects, the complexity of the structures of the brain, and the stringent Blood-Brain Barrier (BBB) that protects the brain from damage and potentially toxic blood-borne molecules. In addition, the lack of efficient technologies to deliver drugs in the deep located and functional brain regions, such as the brain parenchyma, and across the $\mathrm{BBB}$ hinders treatment of brain pathologies $[2,13,14]$. Hence, novel technologies for Glioblastoma cancer therapy must emerge to overcome the BBB blockage while efficiently reaching the brain parenchyma within safety guidelines.

An externally controllable molecular communication platform that consists of stem cells acting as therapeutic, reporting and diagnostic bio-nanomachines has been proposed in the recently granted EU project GLADIATOR: Next-generation Theranostics of Brain Pathologies with Autonomous Externally Controllable Nanonetworks: a Trans-disciplinary Approach with Bio-nanodevice Interfaces (EU-H2020-FET-Open \#828837). The therapeutic bio-nanomachines are autologous organoids of engineered induced Reprogramming Neural Stem Cells (iR-NSCs) implanted into the brain parenchyma to synthesize and release rationally designed therapeutic molecules. The iR-NSCs are controlled by external miniature wearable devices via in-messaging communication channels. Therapeutic molecules collaboratively interfere with the underlying disease pathways in the target Glioblastoma cells. We envision that this proposed technology can overcome the challenges imposed by the BBB and reach the brain parenchyma for the treatment of Glioblastoma cancer. Therapeutic molecules also collaboratively interfere with another type of bio-nanomachines, that we call reporting bio-nanomachines. The reporting bio-nanomachines are engineered Glioblastoma Stem 


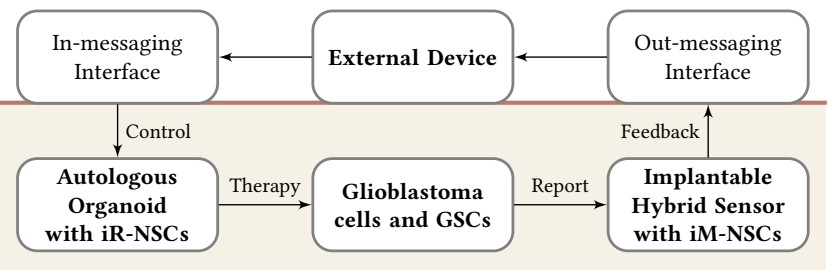

Figure 1: Block diagram of the brain tumour management platform as a fully autonomous externally controllable molecular communication network.

Cells (GSCs) that serve as the gateway for communicating the efficacy of the treatment. Ultimately, the diagnostic bio-nanomachines are engineered induced Monitoring Neural Stem Cells (iM-NSCs) that collect and analyze reporting molecules. The iM-NSCs serve as 'sensors' of the hybrid implantable diagnostic system which provides feedback to external miniature wearable devices via outmessaging communication channels. External wearable devices with enabling communication interfaces, iR-NSCs, GSCs, and iMNSCs form a radically new closed-loop platform for the management of brain malignancies shown in Fig. 1, and provide a breakthrough theranostic (therapeutic + diagnostic) intervention.

A promising strategy for iR-NSCs and GSCs is to use exosomes as cargos to deliver, respectively, therapeutic and reporting molecules to their recipients. Exosomes are $40-100 \mathrm{~nm}$ cell-derived extracellular vesicles that are released from cells upon fusion of an intermediate endocytic compartment, called the multivesicular body, with the plasma membrane [10]. Exosomes play an important role in cell-to-cell signaling containing transmembrane proteins in their lipid bilayer and the cytosol molecular components from their progenitor cell including functional proteins, genetic lipids, genetic materials like messenger RNA (mRNA), microRNA (miRNA), short interfering RNA (siRNA), and genomic DNA (gDNA) [5]. Upon fusion with the target cell, exosomes deliver the transmembrane proteins and biologically active molecules. Due to their biological tolerability, natural targeting, and phagocytosis-inhibition factors, exosomes have been recently regarded as one of the most promising opportunities to deliver chemical packages to the target cell, while protecting the packages from enzymes circulating in body fluids [17]. Hence, exosomes pose as the main information carrier to deliver reactive drug molecules to Glioblastoma cells within a potential therapeutic solution termed as an exosome-mediated drug delivery system [2]. On top of that, the Molecular Communication (MC) ([1]) paradigm becomes the natural choice to characterize, monitor and control the communication system between iR-NSCs, GSCs, and iM-NSCs.

In this paper, we propose an analytical model of the exosomemediated brain drug delivery MC system for analyzing the communication between iR-NSCs and GSCs. By developing an analytical model that characterizes the propagation and reception of the exosomes in the brain parenchyma, we aim to address the quantification of the exosome concentration delivery based on spatial, temporal and frequency analysis. To this end, the considered MC system model encompasses the iR-NSC abstracted as an MC transmitter, the brain extracellular space abstracted as an $M C$ chan$n e l$, and the GSC abstracted as a (reactive) $M C$ receiver, as illustrated

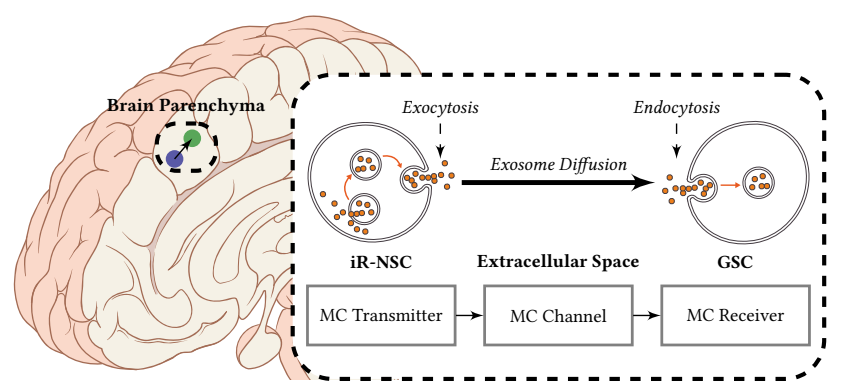

Figure 2: The considered MC system model. iR-NSC is an induced Reprogramming Neural Stem Cell. GSC is a Glioblastoma Stem Cell.

in Fig. 2. Upon reception of external signals, the MC transmitter releases the exosomes with a particular rate through the process called exocytosis. The exosomes then propagate/diffuse through the extracellular space in the brain parenchyma. The exosomes that gather around the $\mathrm{MC}$ receiver bind to the surface of the cell through the process called endocytosis.

Although the MC paradigm has been recently proposed to model particulate drug delivery systems $[6-8,11]$, the existing models do not yield analytical expressions that can be of practical use to model the exosome-mediated brain drug delivery system which has many challenges for maximizing the exosome delivery at cancerous sites. Maximizing the exosome delivery refers to the design and engineering of the exosome structure, mode and location of administration and dosage optimization from iR-NSCs and GSCs. Being the main focus of this paper, proper analytical models of the exosome-mediated MC system and its quantitative analysis are much needed as the first step towards the development of the envisioned Glioblastoma treatment. The contributions are as follows:

- We develop a biophysical analytical model considering the unique targeting properties of exosomes, the unique properties of the brain extracellular space like volume fraction and tortuosity, and the unique exosome uptake mechanism in a form of receptor-mediated endocytosis.

- We derive closed-form solutions for transfer functions of both the channel and receiver, which allow us to conduct a frequency analysis of each module. More specifically, we apply the Fourier transform to the simplified diffusion equation solution in the extracellular space of the brain to derive the channel transfer function; we apply the multi-dimensional Fourier transform and the single-input Volterra series to derive higher order transfer functions for the non-linear endocytosis process at the receiver.

\section{THE EXOSOME-MEDIATED BRAIN DRUG DELIVERY MC SYSTEM MODEL}

The system model located in the brain parenchyma comprises of the one-directional communication system between iR-NSCs (transmitter) and GSCs (receiver) where exosomes serve as information carriers to deliver engineered payload from the IR-NSCs propagating to the brain extracellular matrix (channel). This process is 
depicted in Fig. 2. In the next subsections, we define communication modules from a biophysical perspective that leads to a more detailed description of the functioning and relationship between them.

\subsection{Transmitter}

The exosome emission by the iR-NSCs involves the process of exocytosis where multivesicular bodies are excreted from the intracellularto the extracellular environment. The exocytosis is extremely complex involving numerous intracellular signaling reactions. In this paper, however, we focus on extracellular signaling pathways between bio-nanomachines and exclude the transmitter modeling at this stage. Instead, we consider the result of exocytosis as a fixed excreted concentration of exosomes, $c_{e_{0}}\left(t_{0}, \mathbf{x}_{0}\right)=Q$, where $\mathbf{x}_{0}=\left(x_{0}, y_{0}, z_{0}\right)$ denotes the location of the transmitting iR-NSC.

\subsection{Channel}

The extracellular matrix of the brain is a space where a number of molecules diffuse propagating metabolic information among cells, whether they are neurons or non-neurons. Two main properties of the extracellular matrix in the brain dictate the propagation of molecules, namely volume fraction and tortuosity [21]. We explore these two properties to analyze a channel model for the exosome propagation. We use both volume fraction and tortuosity to generate a model of anisotropic exosome diffusion.

The volume fraction determines a percentage of the total tissue volume accessible to the exosomes. Formally, it is defined as

$$
\alpha=\mathcal{V}_{\text {Extracellular Matrix }} / \mathcal{V}_{\text {Tissue }}
$$

where $\mathcal{V}_{\text {Extracellular Matrix }}$ denotes the volume of the extracellular matrix and $\mathcal{V}_{\text {Tissue }}$ denotes the volume of the whole tissue measure in a small region of the brain. Typically, $\alpha$ can be measured using real-time iontophoresis cation tetramethylammonium (RTI-TMA). Typical values are: $0.1 \leq \alpha \leq 0.3$. Free diffusion medium such as aqueous solutions or very dilute gel can consider $\alpha=1$.

The tortuosity describes the average hindrance of a complex medium relative to an obstacle-free medium. The hindrance results in an effective diffusion which is decreased compared with the free diffusion coefficient of exosomes, $D$. In neurobiological applications, the tortuosity is defined as [21]:

$$
\lambda=\sqrt{D / D^{*}}
$$

where $D^{*}$ is the effective diffusivity of exosomes in the brain extracellular matrix. For an impenetrable medium $\lambda=\infty$; for an obstacle-free medium $\lambda=1$. The tortuosity of the brain extracellular matrix is approximately constant with $\lambda \approx 1$.6. Owing to (2), this means that the exosomes in the brain parenchyma have the effective diffusion coefficient reduced by a factor of $\sim 2.6$ compared with $D$.

We consider $c_{e}(t, \mathbf{x})$ as the concentration of exosomes in the extracellular matrix as a function of both time $t$ and location $\mathbf{x}=$ $(x, y, z)$. Based on the detailed work in [21], we consider the following diffusion equation:

$$
\frac{\partial c_{e}(t, \mathbf{x})}{\partial t}=\underbrace{\frac{D}{\lambda^{2}} \nabla^{2} c_{e}(t, \mathbf{x})+\frac{c_{e_{0}}\left(t_{0}, \mathbf{x}_{0}\right)}{\alpha}}_{\text {diffusion }}-\underbrace{\frac{\left.f\left(c_{e}\right)\right)}{\alpha}}_{\text {uptake }}-\underbrace{\mathbf{v} \nabla c_{e}(t, \mathbf{x})}_{\text {bulk flow }} .
$$

The complicated boundary conditions associated with the intercellular clefts have been replaced by the parameters $\lambda$ and $\alpha$ through the introduction of averaged variables. In (3), the 'diffusion' term represents the propagation of the exosomes, in which the effective diffusion coefficient is defined via tortuosity. The 'uptake' term represents the losses or clearances of material from the extracellular matrix (e.g., into cells, across the BBB, or degradation through enzymatic attack). The $f\left(c_{e}(t, \mathbf{x})\right)$ term represents the Michaelis-Menten update with $f\left(c_{e}(t, \mathbf{x})\right)=-k^{\prime} \alpha c_{e}(t, \mathbf{x})$, where $k^{\prime}$ is a first order scalar defined as $k^{\prime}=\alpha \sigma k$, where $\sigma$ is the ratio of the total surfaces within $\mathcal{V}_{\text {Tissue }}$ to the extracellular volume $\mathcal{V}_{\text {Extracellular Matrix }}$. Either $k^{\prime}$ or $k$ can be obtained through experimental curve fitting approaches [16]. Ultimately, the 'bulk flow' term represents the guiding force upon the exosomes, which is simplified to a generic terms with the velocity vector $\mathbf{v}$ and the subsequent scalar product with the concentration gradient $\nabla c_{e}(t, \mathbf{x})$.

Aiming to account for brain tissue anisotropic properties, (3) is modified by considering the tortuosity as a set of three distinct second-order components, which is possible when considering a natural set of rectangular Cartesian coordinates. Then, we consider the following diffusion equation:

$$
\begin{aligned}
\frac{\partial c_{e}(t, \mathbf{x})}{\partial t} & =\frac{D}{\lambda_{x}^{2}} \frac{\partial^{2} c_{e}(t, \mathbf{x})}{\partial x^{2}}+\frac{D}{\lambda_{y}^{2}} \frac{\partial^{2} c_{e}(t, \mathbf{x})}{\partial y^{2}}+\frac{D}{\lambda_{z}^{2}} \frac{\partial^{2} c_{e}(t, \mathbf{x})}{\partial z^{2}} \\
& +\frac{c_{e_{0}}\left(t_{0}, \mathbf{x}_{0}\right)}{\alpha}-\frac{f\left(c_{e}(t, \mathbf{x})\right)}{\alpha}-\mathbf{v} \nabla c_{e}(t, \mathbf{x})
\end{aligned}
$$

If a signal pulse duration has a value of $t_{p}$, the solution yields by solving (4) due to the linear nature of the diffusion equations and its solutions (see [21]):

$$
c_{e}= \begin{cases}c_{e}(t) & \text { if } t \leq t_{p}, \\ c_{e}(t)-c_{e}\left(t-t_{p}\right) & \text { if } t>t_{p},\end{cases}
$$

with

$$
\begin{aligned}
c_{e}(t, \mathbf{x})= & \frac{Q \lambda_{x} \lambda_{y} \lambda_{z}}{8 \pi D \alpha R}\left[\operatorname{erfc}\left(\frac{R}{2 \sqrt{D t}}+\sqrt{k^{\prime} t}\right) \exp \left(R \sqrt{\frac{k^{\prime}}{D}}\right)+\right. \\
& \left.\operatorname{erfc}\left(\frac{R}{2 \sqrt{D t}}-\sqrt{k^{\prime} t}\right) \exp \left(-R \sqrt{\frac{k^{\prime}}{D}}\right)\right] u(t),
\end{aligned}
$$

where $R=\sqrt{x^{2} \lambda_{x}^{2}+y^{2} \lambda_{y}^{2}+z^{2} \lambda_{z}^{2}}$ and $u(t)$ is the Heaviside function. Assuming $k^{\prime}=0$ and an isotropic propagation where $\lambda_{x}=\lambda_{y}=$ $\lambda_{z}=\lambda, R=r \lambda$ and $r=\sqrt{x^{2}+y^{2}+z^{2}}$, (6) simplifies as:

$$
c_{e}(t, \mathbf{x})=\frac{Q \lambda^{2}}{4 \pi D \alpha r} \operatorname{erfc}\left(\frac{r \lambda}{2 \sqrt{D|t|}}\right) u(t) \text {. }
$$

\subsection{Receiver}

The adhesion of exosomes to the recipient cell utilizes the interaction of various exosomal surface proteins and cellular receptors [15]. Three main mechanisms provide the uptake of exosomes once they bound to the target cell: 1) juxtacrine signaling - where the exosomes elicit transduction via intracellular signaling pathways, 2) fusion - where the exosomes fuse with the cellular membrane and transfer cargo into the cytoplasm, and (iii) endocytosis - where the exosomes internalize and retain in transport vesicles either via 
phagocytosis, macropinocytosis or receptor-mediated endocytosis. Receptor-mediated endocytosis is a specific pattern of particular relevance for the targeted drug delivery systems that reduce the nondiscriminate uptake of exosomes and enhances exosome accumulation at the target site. Receptor-mediated endocytosis takes advantage of differential expression of receptors between disease-affected and normal tissues allowing for a rapid ligandtargeted internalization and controlled intracellular trafficking [22]. For example, the exosomes taken up by clathrin-dependent receptormediated endocytosis are typically destined for lysosomal degradation, unlike clathrin-independent receptor-mediated endocytosis that leads to endosomal accumulation and nondegradation [3]. The general process of receptor-mediated endocytosis is well understood in the literature and involves a sequence of well-orchestrated mechanisms $[12,23]$. In this subsection, we develop the exosome reception model by GSCs from the chemical kinetic model based on a system of ordinary differential equations which describe the temporal evolution of spatially-averaged concentrations of exosomes.

The first step in receptor-mediated endocytosis illustrated in Fig. 3 involves the exosome binding to the target GSC selective receptors in specialized regions of the membrane formed from a protein called clathrin. Clathrin can form lattice-like coats destined for trafficking [19]. The corresponding membrane regions used for endocytosis are accordingly refered to as clathrin-coated pits. Upon binding to clathrin-coated pits, the exosomes become internalized into the GSC cytosol forming endosomes. Recycling/negative feedback mechanisms regulate the number of GSC surface bonds between the exosomes and receptors, leading to the following chemical kinetic model representing the concentration of the exosomes in the brain extracellular matrix $c_{e}(t)$, the concentration of bound exosomes $c_{b}(t)$, and the concentration of internalized exosomes in the GSC cytosol $c_{r}(t)$, respectively [23]:

$$
\begin{aligned}
\beta \frac{\mathrm{d} c_{e}(t)}{\mathrm{d} t} & =\beta k_{e}-a c_{e}(t)\left[p_{m} N(t)+p_{m} N_{0}(t)-c_{b}(t)\right] \\
\frac{\mathrm{d} c_{b}(t)}{\mathrm{d} t} & =a c_{e}(t)\left[p_{m} N(t)+p_{m} N_{0}(t)-c_{b}(t)\right]-k_{i} c_{b}(t) \\
\frac{\mathrm{d} c_{r}(t)}{\mathrm{d} t} & =k_{i} c_{b}(t)-k_{d} c_{r}(t),
\end{aligned}
$$

where $\beta$ is the ratio of the volume of the considered extracellular medium containing $c_{e}$ and the GSC volume, $k_{e}$ is the rate at which the exosomes are supplied to the considered extracellular medium containing $c_{e}, p_{m}$ is the total number of the exosomes that can be bound in the clathrin-coathed pits, $N(t)$ is the total number of occupied clathrin-coathed pits per unit volume at the membrane, $N_{0}(t)$ is the total number of unoccupied clathrin-coathed pits per unit volume at the membrane, $a=a_{0} / p_{m}$ is the rate defined through the maximal binding rate $a_{0}$ measured when none of the exosomes is bound to the membrane, $k_{i}$ is the exosome internalization rate, and $k_{d}$ is the exosome degradation rate.

\section{TRANSFER FUNCTION REPRESENTATION OF THE SYSTEM}

\subsection{Channel Transfer Function}

If a point-source in both space and time is considered where volume $U$ of the exosomes at concentration $C_{e}$ is ejected, then an impulse

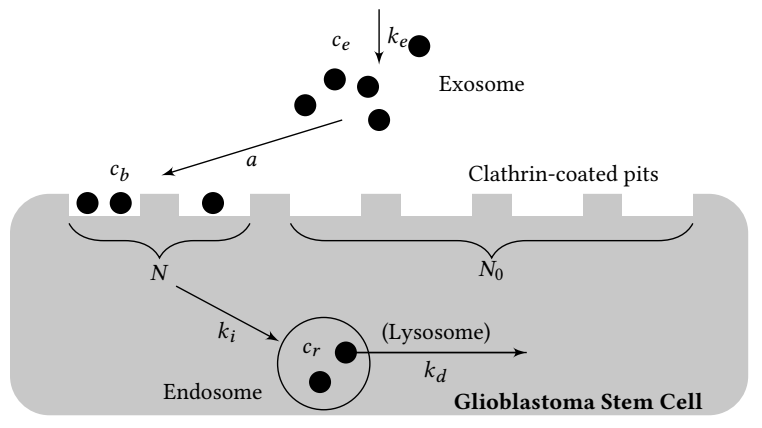

Figure 3: Endocytosis in glioblastoma stem cells.

function solution to (4) follows as [21]:

$$
c_{e}(t, \mathbf{x})=\frac{U C_{e}}{\alpha} \frac{\lambda_{x} \lambda_{y} \lambda_{z}}{(4 \pi D t)^{3 / 2}} \exp \left(-\frac{R^{2}}{4 D t}-k^{\prime} t\right) u(t) .
$$

Assuming $k^{\prime}=0$ and an isotropic propagation, (11) simplifies as:

$$
c_{e}(t, \mathbf{x})=\frac{U C_{e}}{\alpha} \frac{\lambda^{3}}{(4 \pi D t)^{3 / 2}} \exp \left(-\frac{\lambda^{2} r^{2}}{4 D t}\right) u(t) .
$$

Taking the Fourier transform of (12) yields the channel transfer function as:

$$
H_{e}(j \omega)=\int_{-\infty}^{\infty} c_{e}\left(t, \mathbf{x}_{r}\right) \exp (-j \omega t) \mathrm{d} t
$$

where $\mathbf{x}_{r}=\left(x_{r}, y_{r}, z_{r}\right)$ denotes the location of the receiving GSC. This integral is of special form whose solution follows from the identity given in [18, Page 453]:

$$
\begin{aligned}
& \int_{0}^{\infty} x^{\alpha-1} \exp \left(-\frac{q}{x}\right)\left\{\begin{array}{c}
\sin b x \\
\cos b x
\end{array}\right\} \mathrm{d} x=j^{\delta}\left(\frac{q}{b}\right)^{\alpha / 2} \times \\
& {\left[\exp \left(-\frac{\alpha \pi j}{4}\right) K_{\alpha}\left(2 \mathrm{e}^{\pi j / 4} \sqrt{q b}\right) \mp \exp \left(\frac{\alpha \pi j}{4}\right) K_{\alpha}\left(2 \mathrm{e}^{-\pi j / 4} \sqrt{q b}\right)\right]}
\end{aligned}
$$

with $b>0, \operatorname{Re}(q)>0$ and $\operatorname{Re}(\alpha)<1$, where $\delta=\left\{\begin{array}{l}1 \\ 0\end{array}\right\}$ and $K_{\alpha}$ is the modified Bessel function of the second kind (also known as the MacDonald function). By combining (12), (13), and (14), we derive the brain extracellular space channel transfer function as:

$$
H_{e}(j \omega)=2\left(\frac{\lambda^{2} r^{2}}{4 D \omega}\right)^{-1 / 4} \mathrm{e}^{\pi j / 4} K_{-1 / 2}\left(2 \mathrm{e}^{\pi j / 4} \sqrt{\frac{\lambda^{2} r^{2} \omega}{4 D}}\right) .
$$

where

$$
K_{-1 / 2}(x)=\frac{\pi}{2} \frac{I_{1 / 4}(x)-I_{-1 / 4}(x)}{\sin (-\pi / 4)},
$$

where $I_{1 / 4}(x)$ and $I_{-1 / 4}(x)$ are the modified Bessel functions of the first kind. We omit detailed derivation due to the page limit.

\subsection{Receiver Transfer Function}

Combining (8)-(10), we can derive the receiver transfer function with the input to the receiver restricted to follow dynamics defined by (8). However, excluding (8) from the derivation imposes more complicated analysis but offers complete freedom with the input to the receiver. Considering $c_{e}\left(t, \mathbf{x}_{r}\right)$ as the input of the receiver, where $\mathbf{x}_{r}=\left(x_{r}, y_{r}, z_{r}\right)$ denotes the location of the receiving GSC, ensures a concatenation of the channel and receiver transfer functions in 


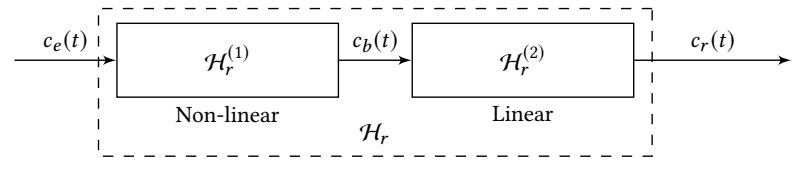

(a) Block diagram for serial combination of non-linear and linear systems.

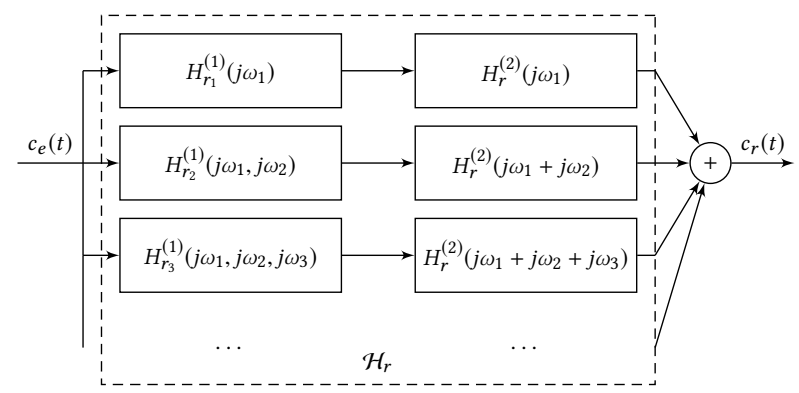

(b) Block diagram for serial combination of Volterra system and linear system.

\section{Figure 4: Block diagram for cascaded model of endocytosis.}

an end-to-end channel analysis that is a natural extension of this work. Thereby, we derive the receiver transfer function from (9) and (10) in what follows.

Eq. (9) that represents dynamics of the concentration of bound exosomes can be simplified by assuming that sum of the total number of occupied clathrin-coated pits per unit volume and the total number of unoccupied clathrin-coated pits per unit volume is constant, i.e., $N(t)+N_{0}(t)=\bar{N}$, where $\bar{N}$ represents the total number of clathrin-coated pits. This is a valid assumption due to receptor dynamics at the membrane [23]. Even though, Eq. (9) remains a non-linear differential equation. Accordingly, the corresponding system $\mathcal{H}_{r}^{(1)}$ in Fig. 4(a) is non-linear.

The single-input Volterra series is well established as a powerful tool in the analysis of non-linear systems [24]. The Volterra series is a generalization of the well-known input-output relation for linear systems, with $h_{1}(\tau), h_{2}\left(\tau_{1}, \tau_{2}\right), h_{3}\left(\tau_{1}, \tau_{2}, \tau_{3}\right), \ldots, h_{n}\left(\tau_{1}, \ldots, \tau_{n}\right)$ representing generalizations of the linear impulse response function called Volterra kernels. The higher order transfer functions or Volterra kernel transforms $H_{n}\left(j \omega_{1}, \ldots, j \omega_{n}\right), n=1, \ldots, \infty$ are defined as the multi-dimensional Fourier transforms. Hence, the following equations apply:

$$
\begin{aligned}
H_{n}\left(j \omega_{1}, \ldots, j \omega_{n}\right)= & \int \cdots \int_{-\infty}^{\infty} h_{n}\left(\tau_{1}, \ldots, \tau_{n}\right) \times \\
& \exp \left(-j\left(\omega_{1} \tau_{1}+\cdots+\omega_{n} \tau_{n}\right)\right) \mathrm{d} \tau_{1} \ldots \mathrm{d} \tau_{n} \\
h_{n}\left(\tau_{1}, \ldots, \tau_{n}\right)= & \frac{1}{(2 \pi)^{n}} \int \cdots \int_{-\infty}^{\infty} H_{n}\left(j \omega_{1}, \ldots, j \omega_{n}\right) \times \\
& \exp \left(j\left(\omega_{1} \tau_{1}+\cdots+\omega_{n} \tau_{n}\right)\right) \mathrm{d} \omega_{1} \ldots \mathrm{d} \omega_{n} .
\end{aligned}
$$

Aiming to determine the analytical form of kernel transforms that we introduce to describe $\mathcal{H}_{r}^{(1)}$, i.e., $h_{r_{1}}^{(1)}(\tau), \ldots, h_{r_{n}}^{(1)}\left(\tau_{1}, \ldots, \tau_{n}\right)$, we apply the method of harmonic probing [4]. In this method, the system is excited by $c_{e}(t)=\exp \left(j \omega_{1} t\right)$ to obtain the first order transfer function $H_{r_{1}}^{(1)}\left(j \omega_{1}\right), c_{e}(t)=\exp \left(j \omega_{1} t\right)+\exp \left(j \omega_{2} t\right)$ to obtain the second order transfer function $H_{r_{2}}^{(1)}\left(j \omega_{1}, j \omega_{2}\right), c_{e}(t)=$ $\exp \left(j \omega_{1} t\right)+\exp \left(j \omega_{2} t\right)+\exp \left(j \omega_{3} t\right)$ to obtain the third order transfer function $H_{r_{3}}^{(1)}\left(j \omega_{1}, j \omega_{2}, j \omega_{3}\right)$, etc., as follows:

$$
\begin{aligned}
H_{r_{1}}^{(1)}\left(j \omega_{1}\right) & =a p_{m} \bar{N} \frac{1}{k_{i}+j \omega_{1}} \\
H_{r_{2}}^{(1)}\left(j \omega_{1}, j \omega_{2}\right) & =-\frac{1}{2} a \frac{H_{r_{1}}^{(1)}\left(j \omega_{1}\right)+H_{r_{1}}^{(1)}\left(j \omega_{2}\right)}{k_{i}+j \omega_{1}+j \omega_{2}} \\
H_{r_{3}}^{(1)}\left(j \omega_{1}, j \omega_{2}, j \omega_{3}\right) & =-\frac{1}{3} a \frac{H_{r_{2}}^{(1)}\left(j \omega_{1}, j \omega_{2}\right)+H_{r_{2}}^{(1)}\left(j \omega_{1}, j \omega_{3}\right)}{k_{i}+j \omega_{1}+j \omega_{2}+j \omega_{3}} \\
& \vdots \\
H_{r_{n}}^{(1)}\left(j \omega_{1}, \ldots, j \omega_{n}\right) & =-\frac{1}{n} a \frac{\sum_{i=1}^{n} H_{r_{n-1}}^{(1)}\left(j \omega_{1}, \ldots, j \omega_{n}\right)}{k_{i}+\sum_{i=1}^{n} j \omega_{i}}
\end{aligned}
$$

$n^{t h}$-order Volterra kernel $h_{r_{n}}^{(1)}\left(\tau_{1}, \ldots, \tau_{n}\right)$ follows by combining (22) and (18).

Eq. (10) that represents dynamics of the concentration of internalized exosomes in the GSC cytosol is linear. Accordingly, the corresponding linear system $\mathcal{H}_{r}^{(2)}$ in Fig. 4(a) can be described with the straightforward transfer function expressed as:

$$
H_{r}^{(2)}(j \omega)=\frac{k_{i}}{k_{d}+j \omega} .
$$

The impulse response $h_{r}^{(2)}(t)$ directly follows as:

$$
h_{r}^{(2)}(t)=\mathcal{F}^{-1}\left\{H_{r}^{(2)}(j \omega)\right\}=k_{i} \exp \left(-k_{d} t\right) u(t) .
$$

The overall transfer function of endocytosis in the target GSC now follows from $H_{r_{n}}^{(1)}\left(j \omega_{1}, \ldots, j \omega_{n}\right)$ given with $(22)$ and $H_{r}^{(2)}(j \omega)$ given with (23), by applying the interconnection cascade law of Volterra series as [20]:

$$
H_{r_{n}}\left(j \omega_{1}, \ldots, j \omega_{n}\right)=H_{r_{n}}^{(1)}\left(j \omega_{1}, \ldots, j \omega_{n}\right) H_{r}^{(2)}\left(j \omega_{1}+\cdots+j \omega_{n}\right),
$$

as shown in Fig. 4(b). The overall impulse response of endocytosis in the target GSC follows by combining (25) and (18) as:

$$
\begin{aligned}
h_{r}\left(\tau_{1}, \ldots, \tau_{n}\right)= & \frac{1}{(2 \pi)^{n}} \int \cdots \int_{-\infty}^{\infty} H_{r_{n}}\left(j \omega_{1}, \ldots, j \omega_{n}\right) \times \\
& \exp \left(j\left(\omega_{1} \tau_{1}+\cdots+\omega_{n} \tau_{n}\right)\right) \mathrm{d} \omega_{1} \ldots \mathrm{d} \omega_{n} .
\end{aligned}
$$

Ultimately, the output endosome concentration follows as Volterra series which express the output of the nonlinear system in "powers" of the input $c_{e}(t)$ as:

$$
c_{r}(t)=\sum_{n=1}^{\infty} \frac{1}{n !} \int_{-\infty}^{\infty} \mathrm{d} \tau_{1} \cdots \int_{-\infty}^{\infty} \mathrm{d} \tau_{n} h_{r}\left(\tau_{1}, \ldots, \tau_{n}\right) \prod_{m=1}^{n} c_{e}\left(t-\tau_{m}\right) .
$$

Note that the first-order kernel $h_{r}\left(\tau_{1}\right)$ is the familiar impulse response of a linear system. The higher order kernels viewed as higher order impulse responses serve to characterize the various orders of nonlinearity in endocytosis. 

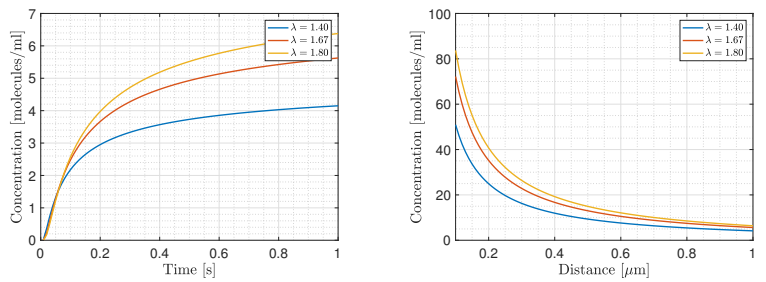

(a) Temporal evaluation of exosomes con- (b) Distance evaluation of exosomes concentrations versus tortuosity variation. centrations versus tortuosity variation.

Figure 5: The effect of tortuosity on the exosome propagation. The tortuosity variability is gathered from the experiments in [21].

\section{NUMERICAL RESULTS}

In this section, we present numerical results in terms of the exosome concentration dynamics, and magnitude and phase for both channel and receiver modules. Since the presented model imposes specific limitations with respect to the whole brain physiological organization and neglect additional interference or noise sources, we provide some remarks about the analysis as follows.

We assume the tortuosity values are known as stationary values. Even though this is true for low $t$ values, tortuosity should be considered as a random variable. For simplicity, we do not include this analysis at this stage.

The channel module is computed by (7) and depicted in Fig. 5(a) and Fig. 5(b). The concentration of exosomes shows a distinct exponential behavior between the temporal and the spatial analysis. By using an initial concentration of $Q=100$ molecules $/ \mathrm{ml}, \alpha=0.2$ and $D=15 \mu \mathrm{m}^{2} / \mathrm{s}$, we observe a continuous increase of exosomes over time with fixed receiver at $1 \mu \mathrm{m}$ apart. The accumulative exosome concentration is different among tortuosity values with the exponential incremental relation leading to a variation of $4-6.4$ molecules/ml. From Fig. 5(a), we infer that higher tortuosity values impose lower loss of the propagating exosomes. From Fig. 5(b), we observe that the concentration of exosomes dramatically decreases with distance increase, converging to near-zero values. This imposes a high impact of molecular diffusion loss in micro-scales in the brain. Even with different tortuosity values, we observe a marginally noticeable difference at $t=1 \mathrm{~s}$. This difference might be significant in large time-scales, potentially more applicable during drug-delivery settings. Since bio-nanomachines are capable of differentiating molecules at very small values, we are looking at an operating range between $0.1-1 \mu \mathrm{m}$ of distance.

The receiver module is computed by (9)-(10) and (27) and depicted in Fig. 6(a) and Fig. 6(b). By using $a=6.64 \times 10^{-17}$ molecule $/ \mathrm{mol} / \mathrm{sec}, N=180, p_{m}=200, k_{i}=0.00271 / \mathrm{s}, k_{d}=0.00021 / \mathrm{s}$ and $c_{e}(t)=1.17 \times 10^{1} 3$ molecule $/ \mathrm{ml}$ [23], we observe an increase over time of $c_{b}(t)$ and $c_{r}(t)$. In particular, while approximately keeping values of $c_{b}(t)=1 \times 10^{4}$, we obtain the increase rate of $8.5 \times 10^{8}$ molecules $/ \mathrm{ml} /$ second. This result tells that the reception process based on $c_{r}(t)$ can be increasingly effective if high concentrations of $c_{e}(t)$ are maintained at stable levels. This is increasingly challenging at realistic settings, but highlights the importance of more complex transmitter designs (not considered in this paper). Ultimately, from

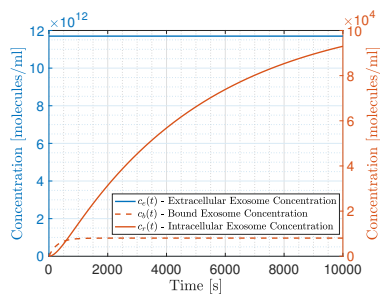

(a) Temporal evaluation of the exosome concentrations based on Eqs. (9-10).

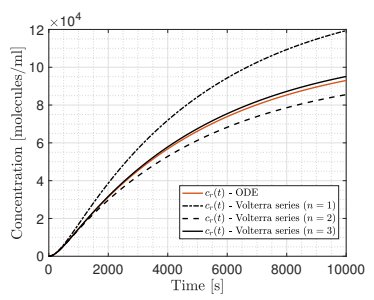

(b) The internalized exosome concentrations based on Eqs. (10) and (27)
Figure 6: Temporal evaluations of the exosome concentrations based on ODEs and Volterra series.
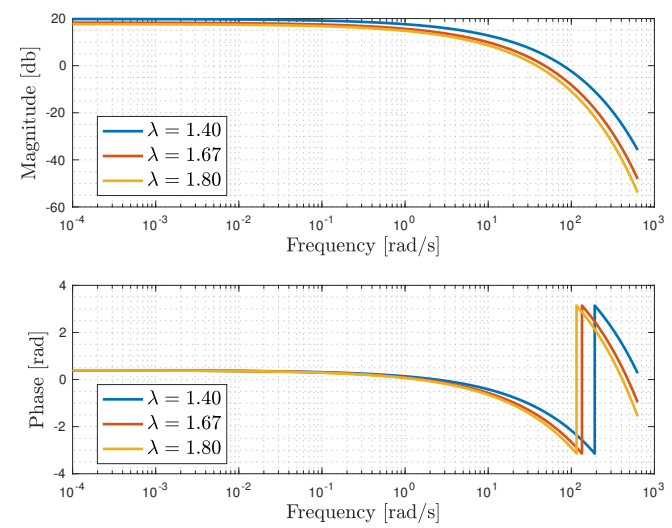

Figure 7: Bode diagram of the channel transfer function $H_{e}(j \omega)$ versus tortuosity variation.

Fig. 6(b) we are able to observe a very good match between a solution of (10) and (27) using $n=3$ Volterra kernels, which indicates the correctness of the analytical approach presented in Section 3.2.

For the frequency analysis, we use the closed-form expressions obtained in (15) and (25) to visualize the corresponding Bode plots. We depict the results in Fig. 7 and Fig. 8. The frequency analysis of the channel module indicates that the effects of tortuosity are frequency dependent for both magnitude and phase. The magnitude drops monotonically for frequencies higher than $100 \mathrm{rad} / \mathrm{s}$, indicating that transmission in these frequencies should be avoided. The phase is approx. $0.35 \mathrm{rad}$ and tortuosity independent for frequencies less than $10 \mathrm{rad} / \mathrm{s}$. Both results, however, converge to the hypothesis that higher levels of tortuosity impact lower operational frequencies. We can observe that the relationship between tortuosity and frequency is favorable to continue with larger tortuosity values. Since the tortuosity measures the level of channel diffusion propagation effectiveness, we can predict that lower tortuosity values will likely have the worst overall communication performance due to its inability to operate in high frequencies.

The frequency analysis of the receiver module is evaluated based on the Volterra kernel transforms using $n=3$. The magnitude of the first-order transfer function $H_{r_{1}}\left(j \omega_{1}\right)$ shown in Fig. 8(a), constantly decreases with the frequency increase, whereas the phase values change between $-\pi$ and $\pi$. The magnitudes of the second-order 

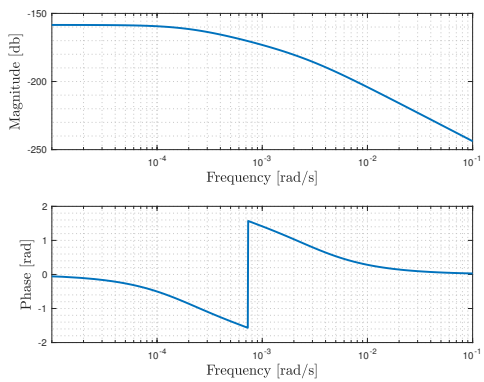

(a) Magnitude and phase of the linear transfer function $H_{r_{1}}\left(j \omega_{1}\right)$.
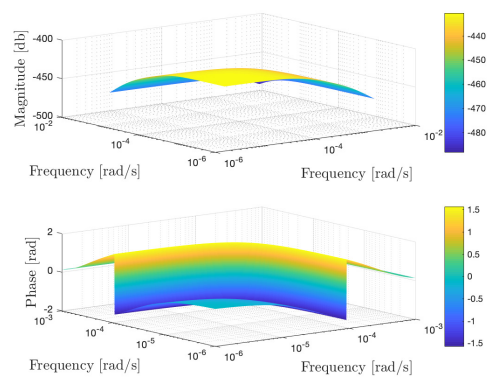

(b) Magnitude and phase of the second-order transfer function $H_{r_{2}}\left(j \omega_{1}, j \omega_{2}\right)$.
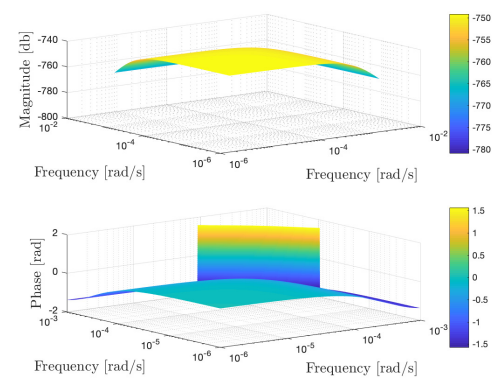

(c) Magnitude and phase of the third-order transfer function $H_{r_{3}}\left(j \omega_{1}, j \omega_{2}, j \omega_{3}=j \omega_{0}\right)$ with $\omega_{0}=2 \pi \mathrm{e}-3$.

Figure 8: Bode diagrams of the receiver Volterra kernel transforms.

transfer function $H_{r_{2}}\left(j \omega_{1}, j \omega_{2}\right)$ and the third-order transfer function $H_{r_{3}}\left(j \omega_{1}, j \omega_{2}, j \omega_{3}=j \omega_{0}\right)$ with $\omega_{0}=2 \pi \times 10^{-3} \mathrm{rad} / \mathrm{s}$ shown in Fig. 8(b) and Fig. 8(c), respectively, both exhibit very low variability and approximates values of $-450 \mathrm{~dB}$ and $-700 \mathrm{~dB}$. Based on these results, the endocytosis perfomed by GSCs shows a very low-frequency operation for a reasonable performance output.

\section{CONCLUSION}

In this paper, we developed a communication engineering model of an exosome-based Molecular Communication (MC) system to support future treatment strategies for Glioblastoma based on externally controlled bio-nanomachines. We present results that show an analysis of the exosome concentration, and Bode diagrams of respective modules (channel and receiver) for an MC system comprising of bio-nanomachines, i.e., engineered stem-cells that emit and collect exosomes that serve as drug cargos. The channel (the extracellular matrix of the brain) shows a dependency on tortuosity which impacts positively with its increase, and a limited operation performance at high frequencies. The receiver (an engineered Glioblastoma stem-cell) shows a dependency on high and stable exosome concentration and low-frequency operation for a reasonable performance output.

Based on the presented models, we foresee continued theoretical developments for maximization of the transmission of exosomes by bio-nanomachines in the brain to control the progression of Glioblastoma and impact negatively in the cancerous cells activity using MC principles. In this way, we believe that these novel models can create better and optimized drug delivery systems based on exosomes by using the newly formed MC knowledge that has been impacting on biotechnology for a number of years now, and continually redefining medicine treatment strategies as we know.

\section{REFERENCES}

[1] I. F. Akyildiz, M. Pierobon, S. Balasubramaniam, and Y. Koucheryavy. 2015. The internet of bio-nano things. IEEE Communications Magazine 53, 3 (2015), 32-40.

[2] C. Altaner and U. Altanerova. 2019. Mesenchymal Stem Cell Exosome-Mediated Prodrug Gene Therapy for Cancer. In Suicide Gene Therapy. Springer, 75-85.

[3] L. Bareford and P. Swaan. 2007. Endocytic mechanisms for targeted drug delivery. Advanced drug delivery reviews 59, 8 (2007), 748-758.

[4] E. Bedrosian and S. O. Rice. 1971. The output properties of Volterra systems (nonlinear systems with memory) driven by harmonic and Gaussian inputs. Proc. IEEE 59, 12 (1971), 1688-1707.
[5] E. J. Bunggulawa, W. Wang, T. Yin, N. Wang, C. Durkan, Y. Wang, and G. Wang. 2018. Recent advancements in the use of exosomes as drug delivery systems. Journal of Nanobiotechnology 16:81, 1 (2018), 1-13.

[6] Y. Chahibi, M. Pierobon, and I. F. Akyildiz. 2015. Pharmacokinetic Modeling and Biodistribution Estimation Through the Molecular Communication Paradigm. IEEE Transactions on Biomedical Engineering 62, 10 (2015), 2410-2420.

[7] Y. Chahibi, M. Pierobon, S. O. Song, and I. F. Akyildiz. 2013. A Molecular Communication System Model for Particulate Drug Delivery Systems. IEEE Transactions on Biomedical Engineering 60, 12 (2013), 3468-3483.

[8] U. A. K. Chude-Okonkwo, R. Malekian, B. T. Maharaj, and A. V. Vasilakos. 2017. Molecular Communication and Nanonetwork for Targeted Drug Delivery: A Survey. IEEE Communications Surveys Tutorials 19, 4 (2017), 3046-3096.

[9] X. Dong. 2018. Current Strategies for Brain Drug Delivery. Theranostics 8, 6 (02 2018), 1481-1493.

[10] James R Edgar. 2016. Q\&A: What are exosomes, exactly? BMC biology 14 (2016), $46 ; 46-46$.

[11] M. Femminella, G. Reali, and A. V. Vasilakos. 2015. A Molecular Communications Model for Drug Delivery. IEEE Transactions on NanoBioscience 14, 8 (2015), 935-945.

[12] M. Gex-Fabry and C. DeLisi. 1984. Model for kinetic and steady state analysis of receptor mediated endocytosis. Mathematical Biosciences 72, 2 (1984), 245-261.

[13] L. I. Goulatis and E. V. Shusta. 2017. Protein engineering approaches for regulating blood-brain barrier transcytosis. Current Opinion in Structural Biology 45 (2017), 109-115.

[14] I. Mäger, A. H. Meyer, J. Li, M. Lenter, T. Hildebrandt, G. Leparc, and M. J. A. Wood. 2017. Targeting blood-brain-barrier transcytosis - perspectives for drug delivery. Neuropharmacology 120 (2017), 4-7.

[15] K. J. McKelvey, K. L. Powell, A. W. Ashton, J. M. Morris, and S. A. McCracken. 2015. Exosomes: Mechanisms of Uptake. Journal of circulating biomarkers 4 (2015), 7; 7-7.

[16] C. Nicholson. 1992. Quantitative analysis of extracellular space using the method of TMA+ iontophoresis and the issue of TMA+ uptake. Canadian journal of physiology and pharmacology 70, S1 (1992), S314-S322.

[17] M. Piffoux, A. K. A. Silva, C. Wilhelm, F. Gazeau, and D. Tareste. 2018. Modification of Extracellular Vesicles by Fusion with Liposomes for the Design of Personalized Biogenic Drug Delivery Systems. ACS Nano 12, 7 (2018), 6830-6842.

[18] A. P. Prudnikov, Yu A. Bryčkov, and O. I. Maric:0Arev. 1981. Integrals and Series of Elementary Functions. Science, Moscow, Russia, Russia.

[19] S. J. Royle. 2006. The cellular functions of clathrin. Cellular and molecular life sciences : CMLS 63, 16 (2006), 1823-1832.

[20] M. Schetzen. 2006. The Volterra and Wiener Theories of Nonlinear Systems. Krieger Publishing Co., Inc., Melbourne, FL, USA.

[21] E. Syková and C. Nicholson. 2008. Diffusion in brain extracellular space. Physiological reviews 88,4 (2008), 1277-1340.

[22] S. Wang, Y. Meng, C. Li, M. Qian, and R. Huang. 2015. Receptor-Mediated Drug Delivery Systems Targeting to Glioma. Nanomaterials (Basel, Switzerland) 6, 1 (2015), 3.

[23] J. A. D. Wattis, B. O’Malley, H. Blackburn, L. Pickersgill, J. Panovska, H. M. Byrne, and K. G. Jackson. 2008. Mathematical Model for Low Density Lipoprotein (LDL) Endocytosis by Hepatocytes. Bulletin of Mathematical Biology 70, 8 (2008), 2303.

[24] K. Worden, G. Manson, and G.R. Tomlinson. 1997. A Harmonic Probing Algorithm for the Multi-Input Volterra Series. Fournal of Sound and Vibration 201, 1 (1997), $67-84$. 